\title{
Protective effect of platelets transfusion during extracorporeal circulation.
}

\author{
Kaiyu Tao', Qi An², Lei Du ${ }^{3}$, Haifeng Cheng ${ }^{1 *}$, Daming Jiang ${ }^{1}$, Zhanglong $\mathrm{Hu}^{1}$ \\ ${ }^{1}$ Department of Cardiovascular Surgery, Second Affiliated Hospital of Zhejiang University School of Medicine, \\ Hangzhou, PR China \\ ${ }^{2}$ Department of Cardiovascular Surgery, West China hospital, Chengdu, PR China \\ ${ }^{3}$ Department of Anesthesiology, West China hospital, Chengdu, PR China
}

\begin{abstract}
Background: Extracorporeal Circulation (ECC) related pulmonary function injury plays an important role in the poor postoperative prognosis or even death in patients undergoing cardiac surgery.

Objective: To investigate whether perioperative administration of platelets can preserve pulmonary function or enhance the inflammatory response and vascular injury.

Methods: Rats were divided into four groups, PreH group, MH group, PostH group and MP group. Neutrophil elastase and Tumor Necrosis Factor- $\alpha($ TNF- $\alpha)$ in rat plasma were detected by ELISA. Oxygenation index and lung water content were measured. Circulating endothelial cells were calculated by flow cytometry and lung tissue pathology was observed. Platelets in the level of systemic inflammatory response, levels of vascular endothelial injury, pulmonary edema and pulmonary function were evaluated.

Results: The level of TNF- $\alpha$ and neutrophil elastase in plasmas were significantly lower in the group that platelets administrated during extracorporeal circulation than that of other groups.

Conclusions: Platelets administrated during the extracorporeal circulation could achieve effect on lung protection and reduce the inflammatory response in plasma. Transfusion of platelets with normal function after rapid consumption might be able to reduce extracorporeal circulation related complications, providing a therapeutic strategy for organ protection during extracorporeal circulation.
\end{abstract}

Keywords: Extracorporeal circulation, Platelets, Lung injury.

Accepted on November 6, 2017

\section{Introduction}

Extracorporeal Circulation (ECC) related pulmonary function injury plays an important role in the poor postoperative prognosis or even death in patients undergoing cardiac surgery [1]. Due to non-physiological contact between blood components and the surface of ECC pipe, hemodynamic changes and low temperature, ECC can lead to the activation of complement system [2], resulting in the activation of inflammatory cells such as neutrophils and monocytes [3], and release of inflammatory factors [4]. These would eventually lead to systemic inflammatory response [5], vascular endothelial cell injury, increased vascular endothelial permeability, and the infiltration of fluid in blood vessels to the pulmonary tissue, resulting in pulmonary edema, reduced compliance and impairment of pulmonary function [6], which manifest as an acute lung injury or even an acute respiratory distress syndrome. Circulating endothelial progenitor cells and adjacent mature endothelial cells were involved in endothelial repair upon endothelial injury [7]. However, it takes a long time to repair endothelial tissues, and it is difficult to complete this within a short period of time $[8,9]$.
Platelets may be the main component involved in endothelial repair [10]. Upon VEC damage, the membrane glycoprotein GPIb-V-IX expressed on the platelet surface binds to the von Willebrand factors (vWFs) in the endothelium and subendothelial layer, causing the rolling and activation of platelets on the blood vessel wall [11]. Activated platelets also express $\beta 1$ integrin and $\beta 3$ integrin (aIIb $\beta 3$, GPIIb/IIIa). The former binds to expose subcutaneous collagen $(\alpha 2 \beta 1)$, fibronectin $(\alpha 5 \beta 1)$ and laminin $(\alpha 6 \beta 1)$, while the latter binds to fibronectin and vWF. These cause platelets to closely bond to VEC and subcutaneous tissues [12], thereby completing the emergency repair of the damaged vascular wall. Uthoff et al. [13] found that the infusion of platelet membrane glycoprotein $\mathrm{IIb} / \mathrm{IIIa}$ (PGIIb/IIIa) antagonists before ECC could reduce platelet depletion during ECC and postoperative blood transfusion. However, it could not improve tissue edema or pulmonary function. In 1999, Madan et al. found that although the use of PGIIb/IIIa antibodies during ECC increased the count of circulating platelets, it also increased postoperative bleeding [14]. Therefore, we can speculate that ECC caused 
endothelial injury, and platelets may be the main component of its early repair.

However, platelet count may be significantly reduced during ECC due to the platelet's surface contact with foreign bodies, activation, adhesion and the use of heparin in ECC [15], especially in the early stage of ECC [16]. Therefore, fresh platelets supplemented in the peri-ECC period could increase normal functional platelet count and further reduce lung injury. This study aimed to investigate the effects of the infusion of platelets on rat systemic inflammatory response, and examine pulmonary function through the peri-ECC infusion of platelets.

\section{Materials and Methods}

\section{Experimental animals and reagents}

A total of 72 male Sprague Dawley (SD) rats (7-8 w of age), weighting 250-300 g, were provided by the Sichuan Provincial Animal Experimental Center. ELISA reagent TNF- $\alpha$ antibody was purchased from Thermo, USA. NE antibody was purchased from USCNLIFE, China. Flow cytometry antibody. CD146 antibody, Phycoerythrin (PE)-conjugated mouse monoclonal anti-rat MCAM/CD146, and CD146 antibody isotype control were purchased from R\&D Systems, USA. VEGFR2 (KDR) antibody was purchased from Novusbio, USA. VEGFR2 antibody isotype control was purchased from GeneTex, USA. FITC-conjugated KDR secondary antibody was purchased from Thermo, USA.

\section{Separation and collection of platelets}

The whole blood collected was immediately used to prepare concentrated platelet plasma after collection according to a reference [16]. The specific steps were as follows: Gradient centrifugation was performed for eight minutes (including 461 $\mathrm{g}$ for four minutes and 2,650 $\mathrm{g}$ for four minutes). The upper plasma was removed together with the buffy coat, a small volume of Red Blood Cells (RBCs) under the buffy coat were placed into another empty blood-collecting tube, and placed at room temperature for one hour. Second gradient centrifugation was performed for six minutes (including $285 \mathrm{~g}$ for two minutes and $461 \mathrm{~g}$ for four minutes). The upper plasma was removed to another blank blood-collecting tube. Finally, plasma was obtained by centrifugation in the second separation, 4,560 $\mathrm{g}$ for six minutes. The upper plasma removed from the blank blood-collecting tubes, and the tubes were frozen at $-20^{\circ} \mathrm{C}$. The remaining $0.5 \mathrm{ml}$ was the concentrated platelet plasma.

Concentrated platelets were prepared on the day of the experiment. Intra-peritoneal injection of $50 \mathrm{mg} / \mathrm{kg}$ of pentobarbital sodium was given for anesthesia. Upon completion of the anesthesia, rats were fixed on a rate plate and exposed with infant laryngoscopy for tracheal intubation (arterial indwelling needle $16 \mathrm{G}$ ). Mechanical ventilation was performed with a small animal ventilator at a respiratory rate of $60 \mathrm{BPM}$, a tidal volume of $15 \mathrm{ml} / \mathrm{kg} / \mathrm{min}$, and an inhaled gas oxygen concentration of $40 \%$. Then, the left ribs of the rats were transected to open the chest and expose the heart. Puncture blood drawing in the right ventricle was performed, and the drawn blood was gently injected into sodium citrate anti-coagulated blood-collecting tubes. Platelet donor rats were sacrificed by overdose anesthesia after blood collection.

\section{Experimental groups}

Rats were divided into MP group (administration with $0.5 \mathrm{ml}$ of plasma during extracorporeal circulation, $n=8$ ), PreH group (administration with $0.5 \mathrm{ml}$ platelet-rich plasma before extracorporeal circulation, $\mathrm{n}=8$ ), $\mathrm{MH}$ group (administration with $0.5 \mathrm{ml}$ platelet-rich plasma during extracorporeal circulation, $\mathrm{n}=8$ ) and PostH group (administration with $0.5 \mathrm{ml}$ platelet-rich plasma after extracorporeal circulation, $n=8$ ).

Another 24 rats were used as platelet donors.

\section{ECC establishment process}

The ECC process performed on rats was according to a previous study [17]. Intra-peritoneal injection of $50 \mathrm{mg} / \mathrm{kg}$ of pentobarbital sodium was performed for anesthesia. Upon completion of anesthesia, rats were fixed on a rate plate and exposed to an infant laryngoscopy fixed to the upper lip for tracheal intubation (arterial indwelling needle $16 \mathrm{G}$ ). Mechanical ventilation was performed with a small animal ventilator at a respiratory rate of $60 \mathrm{BPM}$, a tidal volume of 15 $\mathrm{ml} / \mathrm{kg} / \mathrm{min}$ and an inhaled gas oxygen concentration of $40 \%$. The neck and left thigh root medial skin of rats were disinfected for anatomical exposure of the right common carotid artery and left femoral vein. A $24 \mathrm{G}$ trocar and a tee were implanted in the left femoral vein. Then, a $24 \mathrm{G}$ trocar and a tee were implanted in the right common carotid artery after $10 \mathrm{~min}$ of systematic heparinization $(3 \mathrm{mg} / \mathrm{kg})$ via the left femoral vein. The side opening of the tee was connected to a biological function experimental system to detect arterial blood pressure. Upon successful incubation, all the tubes were sewn to the skin and properly fixed.

\section{Supplementation of platelets for each group}

In PreH group, $0.5 \mathrm{ml}$ of platelets were slowly injected via the side opening of the tee, and the ECC tubes pre-filling at the same time, in order to allow the platelets to mix with the prefilled liquid. In $\mathrm{MH}$ group, $0.5 \mathrm{ml}$ of platelets was slowly injected via the tee piston of the femoral vein at $15 \mathrm{~min}$ after ECC started. For PostH group, $0.5 \mathrm{ml}$ of platelets was slowly injected via the piston at two hours after ECC stopped. In MP group, $0.5 \mathrm{ml}$ of fresh frozen plasma was injected via the tee piston of the femoral vein at 15 min after ECC started.

\section{Specimen and data collection}

Blood gas analysis: At the beginning of ECC, at the end of ECC (two hours after ECC started), and two hours after the end of ECC, arterial blood was collected for blood gas tests (iSTAT); and the pulmonary function of rats was recorded. In addition, appropriate adjustments were made to the ventilator parameters according to arterial carbon dioxide partial 
pressure. Oxygenation index formula: Oxygenation Index $(\mathrm{OI})=\mathrm{PaO}_{2} / \mathrm{FiO}_{2}$ (inhaled oxygen concentration $\mathrm{FiO}_{2}=40 \%$ ).

\section{Blood routine analysis}

For rats in each group, at the beginning of ECC, 15 min after ECC started, 20 min after ECC started, at the end of ECC, and two hours after the end of ECC, approximately $20 \mu \mathrm{l}$ of whole blood was collected from rats in each group for blood tests and recording, and to determine the platelet count and Hematocrit (HCT) levels.

\section{Detection of water content in pulmonary tissues}

Two hours after ECC started, the ribs of rats in each group were transected. The right lungs were ligated and the pulmonary tissue was cut off at the distal end along the ligation line. The liquid on the surface of the pulmonary tissue was gently wiped with a cotton swab, and was weighed and recorded. Then, the pulmonary tissue was placed in a dry oven at $60^{\circ} \mathrm{C}$ for $72 \mathrm{~h}$ and weighed. Water content in the pulmonary tissue was calculated with the following formula to evaluate pulmonary tissue edema: water content in pulmonary tissue $=($ wet weight of pulmonary tissue-dry weight of pulmonary tissue)/wet weight of pulmonary tissue $\times 100 \%$.

\section{Detection of inflammatory factors (TNF- $\alpha$ and NE)}

Blood was drawn from rats of each group, and then gently injected into EDTA anti-coagulated blood-collecting tubes prior to ECC $(0.5 \mathrm{ml}$, in order to reduce pre-ECC blood loss), at the end of ECC $(1 \mathrm{ml})$, and two hours after ECC $(1 \mathrm{ml})$. The collected blood was immediately centrifuged $\left(4^{\circ} \mathrm{C}, 3,500\right.$ RPM, $10 \mathrm{~min}$ ). The supernatant was dispensed in two EP tubes and placed in a refrigerator at $-70^{\circ} \mathrm{C}$ for cryopreservation, in order to determine the plasma levels of inflammatory factors. The concentration of TNF- $\alpha$ and NE was determined by ELISA.

\section{Flow cytometry detection of circulating endothelial cells}

For circulating endothelial cells, CD146+ and KDR+ were used as screening indicators. Double positive cells were identified as circulating endothelial cells. The operation was performed according to the instructions of the antibodies.

\section{Pathological examination of pulmonary tissues}

Two hours after the end of ECC, the pulmonary tissue of rats in each group was dissected. The ribs of rats were transected and the right lungs were ligated. Then, the pulmonary tissue was cut off at the distal end along the ligation line and stored immediately in $4 \%$ paraformaldehyde to prepare the sections for hematoxylin and eosin ( $\mathrm{H}$ and $\mathrm{E})$ staining.

\section{Statistical analysis}

Homogeneity test of variance was performed for the data of each group prior to comparison. Further variance analysis would be performed for homogeneous data, while variance change would be of necessity for heterogeneous analysis prior to further analysis. Analysis was carried out using software: SPSS 16.0 for windows (SPSS Inc., Chicago, IL, USA). A P value $<0.05$ was considered statistically significant.

\section{Results}

\section{General situation}

There was no significant difference in the body weight of rats in all groups. Except MH group, there was one rat died in each of the other groups. The cause of death was operative field bleeding and insufficient intraoperative anesthesia, resulting in limb movement and needle falling out. During the experiment, an additional rat was included in PreH group, MP group and PostH group to maintain eight rats in each group. Platelet concentration was $7,853.58 \pm 849.65 \times 10^{9} / \mathrm{L}$, platelet harvest rate was $61.2 \pm 7.6 \%$, the count of mixed White Blood Cells (WBCs) was $5.06 \pm 0.35 \times 10^{8} / \mathrm{L}$, and the count of mixed RBCs was $11.7 \pm 1.6 \times 10^{9} / \mathrm{L}$. The platelet harvest rate reached the criteria of the American Association of Blood Bank $(\mathrm{AABB})$ standard [18], that is, a platelet harvest rate of $60 \%$.

\section{Platelet count in blood of rats of each group}

The platelet count of rats in $\mathrm{PreH}$ group was significantly higher than that in the other groups $(\mathrm{P}<0.001)$ (Figure 1A). The platelet count in MP group began to drop from the beginning of ECC and in two hours after the end of ECC $(\mathrm{P}<0.001)$. The circulating platelet count of rats in each group significantly increased after transfusion of platelets. The platelet count in $\mathrm{MH}$ group increased from $811.2 \pm 189.1 \times 10^{9} / \mathrm{L}(20 \mathrm{~min}$ after the beginning of ECC) to $547.5 \pm 109.6 \times 10^{9} / \mathrm{L}$ (prior to supplementation of platelets, $15 \mathrm{~min}$ after the beginning of ECC), and there was no significant difference compared with that at the beginning of ECC $\left(894.8 \pm 61.7 \times 10^{9} / \mathrm{L}, \mathrm{P}=0.525\right)$. However, there was a significant difference of the platelet count in PostH group upon supplementation of platelets after the end of ECC, compared with that at $15 \mathrm{~min}$ after the beginning of ECC, 20 min after the beginning of ECC, and at the end of ECC $(p<0.01)$. Rats in MH group supplemented with platelets at $20 \mathrm{~min}$ after the end of ECC also had a significantly higher count than the other groups $(p<0.05)$. Furthermore, rats in PostH group had a significantly higher count than rats in MP group $(p<0.001)$ and $\mathrm{PreH}$ group $(\mathrm{P}=0.001)$. This suggests that the transfusion of platelets during ECC can significantly increase the count of circulating platelets.

\section{Blood gas of rats in each group}

There was no significant difference of oxygenation index among these groups at the beginning of ECC (Figure 1B). Compared with the beginning of ECC, the arterial oxygenation index of rats significantly decreased in two hours after the end of ECC in all groups $(\mathrm{p}<0.05)$. Among these groups, PreH group had the most obvious decrease. These levels were significantly lower at two hours after the end of ECC than at 
the beginning of ECC $(p<0.001)$ and at the end of ECC $(p<0.05)$. In MP group, these levels were also significantly lower at two hours after the end of ECC than at the beginning of ECC $(p<0.001)$ and at the end of ECC $(p<0.05)$. The oxygenation index decrease in $\mathrm{MH}$ group was the smallest, which decreased from $380.5 \pm 35.0 \mathrm{mmHg}$ (at the beginning of ECC) to $326.6 \pm 39.9 \mathrm{mmHg}$ (two hours after the end of ECC) $(\mathrm{P}=0.015)$. There was no significant difference between oxygenation indexes at the end of ECC and at two hours after the end of ECC in each group.

\section{Water content in pulmonary tissues among rats at $2 \mathrm{~h}$ after the end of ECC}

Since the water content of pulmonary tissues could not be detected before the beginning of ECC, the water content of pulmonary tissues was detected at two hours after the end of ECC. MH group had the least water content, which was $79.0 \pm$ $1.4 \%$. PostH group had the highest water content in pulmonary tissues $(80.0 \pm 1.8 \%)$. There was no significant difference among these groups $(\mathrm{P}>0.05)$ (Figure 1C).

\section{TNF- $\alpha$ levels and NE levels measurement}

There was no significant difference in plasma TNF- $\alpha$ level among these groups at the beginning of ECC (Figure 1D). Along with the time axis, the levels of TNF- $\alpha$ in the plasma of rats in each group were significantly higher than those at the beginning $(\mathrm{P}<0.01)$. Rats in MP group had significantly lower levels at two hours after the end of ECC than that at the end of ECC $(\mathrm{P}<0.01)$. Furthermore, rats in $\mathrm{MH}$ group had significantly lower levels at two hours after the end of ECC than that at the end of ECC $(\mathrm{P}<0.01)$.

At the end of ECC, plasma TNF- $\alpha$ level was the highest in PreH group, but there was no significant difference compared with that in MP group $(\mathrm{P}=0.365)$ and $\mathrm{PostH}$ group $(\mathrm{P}=0.55)$. Plasma TNF- $\alpha$ level was lowest in $\mathrm{MH}$ group, which was significantly lower than that in $\operatorname{PreH}$ group $(\mathrm{P}=0.011)$. At two hours after the end of ECC, plasma TNF- $\alpha$ level was the highest in $\mathrm{PreH}$ group, but there was no significant difference when compared with MP group $(\mathrm{P}=0.213)$ and $\mathrm{PostH}$ group $(\mathrm{P}=0.577)$. Plasma TNF- $\alpha$ level was the lowest in $\mathrm{MH}$ group, which was significantly lower than that in $\mathrm{MP}$ group $(\mathrm{P}=0.04)$, PreH group $(\mathrm{P}<0.001)$ and $\mathrm{PostH}$ group $(\mathrm{P}=0.003)$. It was indicated that the infusion of large doses of platelets during ECC can reduce the level of TNF- $\alpha$ in circulation after the end of ECC. There was no significant difference in plasma NE level among groups at the beginning of ECC (Figure 1E). With the time axis, the levels of NE in plasma in each group were significantly higher than those at the beginning of ECC $(\mathrm{P}<0.01)$. Among these, MP group had significantly higher levels at two hours after the end of ECC than that at the beginning of ECC and at the end of $\mathrm{ECC}(\mathrm{P}<0.01)$.

At the end of ECC, plasma NE level was the lowest in $\mathrm{MH}$ group, which was significantly lower than that in PostH group $(\mathrm{P}=0.002)$. NE level was the lowest in $\mathrm{MH}$ group at two hours after the end of ECC, which was significantly lower than that in MP group $(\mathrm{P}=0.009)$ and $\mathrm{PostH}$ group $(\mathrm{P}=0.039)$. It was indicated that the infusion of ECC during ECC can relieve the activation of neutrophil in circulation.

\section{Circulating endothelial cell counts at the end of ECC in each group}

Considering the excessive blood loss due to multiple checks at the beginning of ECC, circulating endothelial cell count was not detected at the beginning of ECC. However, this was detected at the end of ECC (Figure 1F). There was no significant difference in circulating endothelial cell count among these groups.

\section{Pathological comparison of inflammatory tissues of rats at $2 \mathrm{~h}$ after the end of $E C C$}

The HE pathological sections of pulmonary tissues were compared. Tissues were thinnest and the infiltration of inflammatory cells was the least in $\mathrm{MH}$ group. Pulmonary tissues were thickest in $\mathrm{PreH}$ and PostH groups, which indicated severer pulmonary edema (Figure 2).

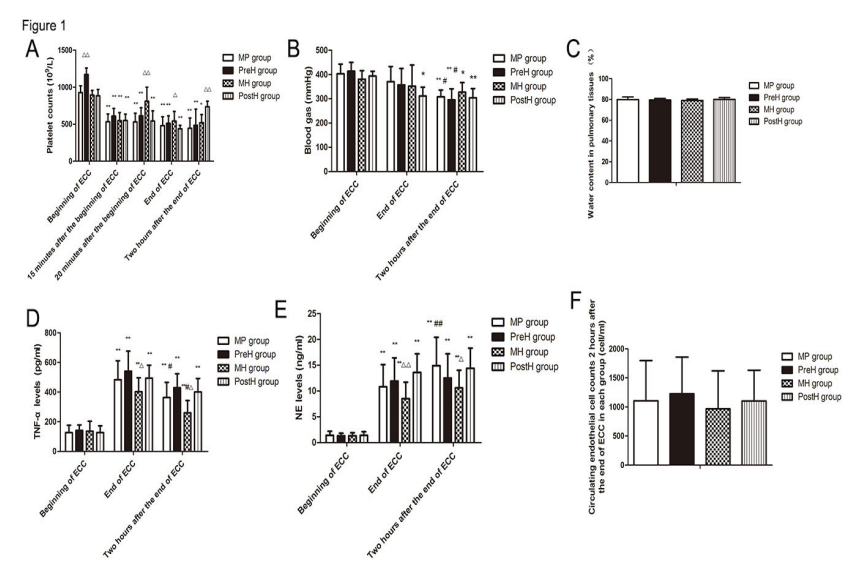

Figure 1. Data measurement. A. Comparison of platelet counts in the blood of rats in the MP, PreH, MH and PostH groups at different time points $\left(10^{9} / \mathrm{L}\right)$. For intra-group comparison with the beginning of ECC, ${ }^{*} P<0.05,{ }^{* *} P<0.01$; for intra-group comparison at 15 min after the beginning of ECC, at 20 min after the beginning of ECC, and at the end of ECC, ${ }^{\#} P<0.01$; for comparison among groups at the same time point, ${ }^{\Delta} P<0.05 ;{ }^{\Delta}{ }^{\Delta}<0.01$. B. Blood gas comparison of rats in $\mathrm{MP}$, PreH, MH, and PostH groups at different time points $(\mathrm{mmHg})$. For intra-group comparison with the beginning of ECC, ${ }^{*} P<0.05$; ${ }^{* *} P<0.01$; for intra-group comparison at the end of $E C C,{ }^{\#} P<0.05$. $C$. Comparison of water content in pulmonary tissues among rats in the MP, PreH, MH and PostH groups at two hours after the end of ECC (\%). D. Comparison of plasma TNF-a levels in rats in the four groups $(\mathrm{pg} / \mathrm{ml})$. For intra-group comparison with the beginning of ECC, ${ }^{* *} P<0.01$; for intra-group comparison at the end of ECC, ${ }^{\#} P<0.05$; for comparison among groups at the same time point, ${ }^{\Delta} P<0.05$. E. Comparison of plasma NE levels of rats in the $M P$, PreH, MH and PostH groups at different time points $(\mathrm{ng} / \mathrm{ml})$. For intra-group comparison with the beginning of ECC, ${ }^{* *} P<0.01$; for intra-group comparison at the end of $E C C,{ }^{\#} P<0.01$; for comparison among groups at the same time point, ${ }^{\Delta} P<0.05 ;{ }^{\Delta} P<0.01 . \quad F$. Comparison of circulating endothelial cell counts at the end of ECC in each group $(\mathrm{cell} / \mathrm{ml})$. 


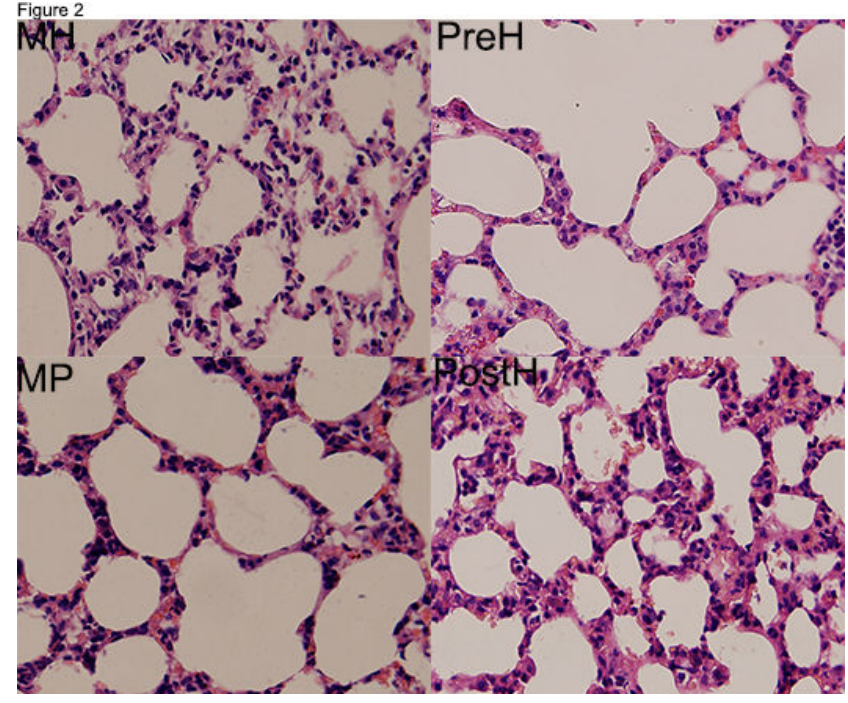

Figure 2. Pathological comparison of inflammatory tissue of rats in the MP, PreH, MH and PostH groups at two hours after the end of ECC (X400).

\section{Discussion}

Low temperature [19], non-physiological contact with the tube surface [20] and heparin [21] in ECC were considered to have the ability to activate platelets. Activated platelets attach to the non-physiological surface of ECC tubes, adhered to the vascular endothelial layer, adhered to the sub-endothelial matrix via vWFs and released inflammatory factors. Activated endothelial cells release chemotactic factors such IL-8, causing the further chemo taxis of neutrophils, and promotes neutrophil adhesion, infiltration and retention in tissues. Therefore, it is considered that during ECC, it is necessary to inhibit the activity of platelets and reduce its activation and adhesion. Tirofiban [22], abciximab [23], FK633 (short-acting GPIIb/IIIa receptor inhibitors) [24] and others have been applied during ECC, which mainly inhibit the main activated receptor GPIIb/ IIIa for adhesion on the surface of platelets, and lower platelet depletion. However, there are contrary conclusions on postoperative bleeding by the use of GPIIb/IIIa receptor inhibitors [13,14,22]. Activated platelets could increase the expression of $\beta 1$ integrin and $\beta 3$ integrin (aIIb $\beta 3$, GPIIb/IIIa). These lead to the close binding of platelets to VEC and subcutaneous tissues [12], thereby completing the emergency repair of the damaged vascular wall. Activated platelets, as well as proteins and lipids released by platelets, can maintain the integrity of microcirculation [25-29]. The reduction in circulating platelet count would lead to increased fluid exudation and tissue edema [30].

ECC can cause systemic inflammatory response and activate vascular endothelial cells, resulting in the contracture and deformation of vascular endothelial cells, increased cell gap, and even the peeling off of vascular endothelial cells, in which causes a significant increase in capillary permeability [31]. The exposure of subcutaneous collagen tissues further promotes the adhesion and infiltration of inflammatory cells such as neutrophils. The release of inflammatory factors may cause tissue damage and intensified tissue edema. It has been reported that capillary leakage due to ECC-related inflammatory response was the main cause for pulmonary dysfunction after ECC [32]. However, during ECC, the nonphysiological contact with tubes caused the activation and adhesion of a large amount of platelets, causing the platelet count to significantly decrease. It was reported in a literature that at five minutes after the beginning of ECC, platelet count can be reduced to less than $20 \%$ of the normal count [33]. In addition, during ECC, platelet aggregation and adhesion functions were severely impaired, which greatly reduced its role in repairing the vascular endothelium [34]. These hindered platelets from being involved in the repair of the vascular endothelium during ECC, aggravating the severity of ECCrelated organ damage.

According to the above evidence, fresh platelets supplemented to ECC may strengthen the role of platelets in repairing the vascular endothelium. Considering that there is no blood transfusion rejection between allogeneic rats at the first blood transfusion [35]. In this study, fresh palates were infused to allogeneic rats at different time points to investigate the effects of elevated platelet counts at different time points on the level of inflammatory response in rats, as well as its impact on pulmonary function. At $15 \mathrm{~min}$ after the start of ECC, platelet count (corrected with HCT) was rapidly reduced to $60.4 \pm$ $2.4 \%$ of the level at the beginning of ECC, and over two thirds of platelets were lost due to the contraction of blood components with ECC foreign bodies. Platelet count significantly increased in five minutes after the supplementation of platelets to the ECC, and there was no significant difference in platelet count compared with the level at the beginning of ECC. This indicates that the use of concentrated platelet preparations in this study could significantly increase the count of platelets in circulation. Although the supplementation of fresh platelets to ECC did not show a significant protection for pulmonary function in two hours after ECC circulation, it could significantly improve the level of inflammatory response in circulation. We found that the levels of TNF- $\alpha$ and NE in the circulation of plateletsupplemented rats after $15 \mathrm{~min}$ of in vitro circulation were significantly lower than those in the control group and the other groups with platelets supplemented at different time. TNF- $\alpha$ is secreted by activated macrophages, monocytes and neutrophils, which are the earliest and most important endogenous inflammatory mediators in inflammatory response [36]. NE is mainly secreted by neutrophils, and is a member of the serine protease family. In the physiological state, NE participates in the body defense system to kill pathogens and protect the body from infections. However, in pathological conditions, ECC, NE is largely secreted, which can destroy cell tight junctions and the integrity of the basement membrane, resulting in increased pulmonary vascular permeability and pulmonary edema, and further causing inflammatory injury in pulmonary tissues [37]. Previous research has showed that the activation level of NE is closely related to pulmonary tissue injury [38]. This indicates that the supplementation of fresh platelets to the ECC can significantly reduce the activation of 
inflammatory cells in circulation. The pathological sections of pulmonary tissues also suggest that the supplementation of platelets to the ECC can significantly reduce pulmonary edema, the retention of neutrophils and other inflammatory cells in pulmonary tissues. A recent study has also suggested that platelets can protect lung from injury induced by systemic inflammatory responses. ECC would causes systemic inflammation and pulmonary dysfunction, and platelet transfusion resulted in significantly milder lung injury and higher lung function. Platelet transfusion was associated with higher production of transforming growth factor- $\beta$ and as well as lower levels of tumour necrosis factor- $\alpha$ and neutrophil elastase in plasma and lung [39].

Our study has also found that the pre-filling of platelets prior to the start of ECC can significantly increase platelet count, but does not reduce systemic and pulmonary inflammatory response, or significantly increase this response, compared with rats supplemented with platelets during ECC. The infusion of platelets during ECC plays a certain role in lung protection. This indicates that platelets supplemented prior to ECC may cause more platelets in circulation to get in contact with the ECC tube surface for adhesion and activation. The more platelets involved in inflammatory response are and the more platelets would be consumed, which causes less platelets to be involved in vascular endothelial repair. Therefore, this manifests as proinflammatory response.

In conclusion, the inflammatory response levels detected at different time points of platelet transfection in rats suggest that platelets in ECC not only participate in inflammatory response, but are also involved in anti-inflammatory response. At different time points, platelets play a different role in proinflammatory response and anti-inflammatory response. Furthermore, if a certain amount of platelets were maintained for normal adhesion and aggregation during ECC, they would reduce the incidence of ECC-related complications and improve the prognosis.

\section{Acknowledgment}

This study was supported by Zhejiang Provincial Natural Science Foundation of China, no. LQ13H020002.

\section{Conflict of Interest}

There is no conflict of interest of all authors.

\section{References}

1. Christian FS, Lara SS. Recent developments in the perioperative management of the paediatric cardiac patient. Curr Opin Anaesthesiol 2006; 19: 375-381.

2. Nilsson B, Ekdahl KN, Mollnes TE, Lambris JD. The Role of Complement in Biomaterial induced Inflammation. Mol Immunol 2007; 44: 82-94.

3. Edmunds LH. Inflammatory response to cardiopulmonary bypass. Ann Thorac Surg 1998; 66: 12-16.
4. Lappegard KT, Bergseth G, Riesenfeld J, Pharo A, Magotti $\mathrm{P}$, Lambris JD, Mollnes TE. The artificial surface-induced whole blood inflammatory reaction revealed by increases in a series of chemokines and growth factors is largely complement dependent. J Biomed Mater Res A 2008; 87: 129-135.

5. Sablotzki A, Friedrich I, Muhling J, Dehne MG, Spillner J, Silber RE, Czeslik E. The systemic inflammatory response syndrome following cardiac surgery: different expression of proinflammatory cytokines and procalcitonin in patients with and without multiorgan dysfunctions. Perfusion 2002; 17: 103-109.

6. Apostolakis E, Filos KS, Koletsis E, Dougenis D. Lung dysfunction following cardiopulmonary bypass. J Card Surg 2010; 25: 47-55.

7. Bai X, Wang $X, X u$ Q. Endothelial damage and stem cell repair in atherosclerosis. Vascul Pharmacol 2010; 52: 224-229.

8. Xu Q. The impact of progenitor cells in atherosclerosis. Nat Clin Pract Cardiovasc Med 2006; 3: 94-101.

9. Xu Q, Zhang Z, Davison F, Hu YH. Circulating progenitor cells regenerate endothelium of vein graft atherosclerosis, which is diminished in ApoE-deficient mice. Circ Res 2003; 93: 76-86.

10. Varga-Szabo D, Pleines I, Nieswandt B. Cell adhesion mechanisms in platelets. Arterioscler Thromb Vasc Biol 2008; 28: 403-412.

11. Offermanns S. Activation of platelet function through $G$ protein-coupled receptors. Circ Res 2006; 99: 1293-304.

12. Ruggeri ZM. Structure and function of von Willebrand factor. Thromb Haemost 1999; 82: 576-584.

13. Uthoff K, Zehr KJ, Geerling R, Herskowitz A, Cameron DE, Reitz BA. Inhibition of platelet adhesion during cardiopulmonary bypass reduces postoperative bleeding. Circulation 1994; 90: 269-274.

14. Madan M, Blankenship JC, Berkowitz SD. Bleeding complications with platelet glycoprotein IIb/IIIa receptor antagonists. Curr Opin Hematol 1999; 6: 334-341.

15. Paparella D, Brister SJ, Buchanan MR. Coagulation disorders of cardiopulmonary bypass: a review. Intensive Care Med 2004; 30: 1873-1881.

16. Hirosue A, Yamamoto K, Shiraki H, Kiyokawa H, Maeda Y, Yoshinari M. Preparation of white-cell-poor blood components using a quadruple bag system. Transfusion 1988; 28: 261-264.

17. Xie XJ , Tao KY, Tang ML, Du L, An Q, Lin K, Gan $\mathrm{CP}$, Chen YW, Luo SH. Establishment of rat extracorporeal circulation model and evaluation on its effects. J Sichuan Univ (Medicine Edn.) 2012; 43: 770-774.

18. Schmldt PT. Standards for blood banks and transfusion services. QRB Qual Rev Bull 1977; 3: 17-22.

19. Boldt J, Knothe C, Welters I, Dapper FL, Hempelmann G. Normothermic versus hypothermic cardiopulmonary bypass: do changes in coagulation differ? Ann Thorac Surg 1996; 62: 130-135. 
20. Gemmell CH, Ramirez SM, Yeo EL, Sefton MV. Platelet activation in whole blood by artificial surfaces: identification of platelet-derived microparticles and activated platelet binding to leukocytes as material-induced activation events. J Lab Clin Med 1995; 125: 276-287.

21. Gouin I, Lecompte T, Morel MC, Lebrazi J, Modderman PW, Kaplan C, Samama MM. In vitro effect of plasmin on human platelet functions in plasma. Inhibition of aggregation caused by fibrinogenolysis. Circulation 1992; 85: 935-941.

22. Bizzarri F, Scolletta S, Tucci E, Lucidi M, Davoli G, Toscano T, Neri E, Muzzi L, Frati G. Perioperative use of tirofiban hydrochloride (Aggrastat) does not increase surgical bleeding after emergency or urgent coronary artery bypass grafting. J Thorac Cardiovasc Surg 2001; 122: 1181-1185.

23. Dyke C, Bhatia D. Inhibitors of the platelet receptor glycoprotein IIb-IIIa and complications during percutaneous coronary revascularization. Management strategies for the cardiac surgeon. J Cardiovasc Surg 1999; 40: 505-516.

24. Kondo N, Suzuki Y, Wakayama F, Tamai Y, Ji K, Fukui K, Fukuda I. Platelet preservation with a glycoprotein IIb/IIIa inhibitor in a porcine cardiopulmonary bypass model. Ann Thorac Surg 2005; 80: 251-257.

25. Gimbrone MA, Aster RH, Cotran RS, Corkery J, Jandl JH, Folkman J. Preservation of vascular integrity in organs perfused in vitro with a platelet-rich medium. Nature 1969. 221: 33-36.

26. Garcia JG, Liu F, Verin AD, Birukova A, Dechert MA, Gerthoffer WT, Bamberg JR, English D. Sphingosine 1phosphate promotes endothelial cell barrier integrity by Edg-dependent cytoskeletal rearrangement. J Clin Invest 2001; 108: 689-701.

27. Schaphorst KL, Chiang E, Jacobs KN, Zaiman A, Natarajan V, Frederick W, Joe GNG. Role of sphingosine-1 phosphate in the enhancement of endothelial barrier integrity by platelet-released products. Am J Physiol Lung Cell Mol Physiol 2003; 285: 258-267.

28. Dudek SM, Jacobson JR, Chiang ET, Birukov KG, Wang P, Zhan X, Garcia JG. Pulmonary endothelial cell barrier enhancement by sphingosine 1-phosphate: roles for cortactin and myosin light chain kinase. J Biol Chem 2004; 279: 24692-24700.

29. Zhang G, Xu S, Qian Y, He P. Sphingosine-1-phosphate prevents permeability increases via activation of endothelial sphingosine-1-phosphate receptor 1 in rat venules. Am J Physiol Heart Circ Physiol 2010; 299: 1494-504.
30. Shepro, D, Welles, SL, Hechtman, HB. Vasoactive agonists prevent erythrocyte extravasation in thrombocytopenic hamsters. Thromb Res 1984; 35: 421-430.

31. Royston D, Minty BD, Higenbottam TW, Wallwork J, Jones GJ. The effect of surgery with cardiopulmonary bypass on alveolar-capillary barrier function in human beings. Ann Thorac Surg 1985; 40: 139-143.

32. Brudney CS, Gosling P, Manji M. Pulmonary and renal function following cardiopulmonary bypass is associated with systemic capillary leak. J Cardiothorac Vasc Anesth 2005; 19: 188-192.

33. Tabata S1, Yamaguchi S, Nagamine H,Tomita S, Arai S, Takemura H, Watanabe G. Efficacy of FK633, an ultrashort acting glycoprotein IIb/IIIa antagonist on platelet preservation during and after cardiopulmonary bypass. Eur J Cardiothorac Surg 2004; 26: 289-293.

34. Linden MD. The hemostatic defect of cardiopulmonary bypass. J Thromb Thrombolysis 2003; 16: 129-147.

35. Hongbin A, Jing Z, Huiyuan F. Comparative studies on blood types of several kinds of vertebrates. Shandong Norm Univ (Nat Sci) 2002; 17: 67-70.

36. Celik JB, Gormus N, Okesli S, Gormus ZI, Solak H. Methylprednisolone prevents inflammatory reaction occurring during cardiopulmonary bypass: effects on TNFalpha, IL-6, IL-8, IL-10. Perfusion 2004; 19: 185-191.

37. Ishii $\mathrm{T}$, Doi $\mathrm{K}$, Okamoto $\mathrm{K}$, Imamura $\mathrm{M}$, Dohi $\mathrm{M}$, Yamamoto K, Fujita T, Noiri E. Neutrophil elastase contributes to acute lung injury induced by bilateral nephrectomy. Am J Pathol 2010; 177: 1665-1673.

38. Tamakuma S, Ogawa M, Aikawa N, Kubota T, Hirasawa H, Ishizaka A, Taenaka N, Hamada C, Matsuoka S, Abiru T. Relationship between neutrophil elastase and acute lung injury in humans. Pulm Pharmacol Ther 2004; 17: 271-279.

39. Luo S, Wang Y, An Q, Chen H, Zhao JF, Zhang J, Meng WT, Du L. Platelets protect lung from injury induced by systemic inflammatory response. Sci Rep 2017; 7: 42080.

\section{*Correspondence to}

Haifeng Cheng

Department of Cardiovascular Surgery

Second Affiliated Hospital of Zhejiang University School of Medicine

PR China 